\title{
Proposal for a Random-based Algorithm to Solve Data-fitting Problems
}

\author{
Giorgio Gambirasio (giga@uol.com.br)
}

\begin{abstract}
This preprint is a complement to a preceding article. Since error-squaring exaggerates the influence of outliers in the linear least square method, a more natural procedure to avoid mutual cancellation of errors would be taking absolute values of errors instead. However, such a choice would prevent using differential calculus. It is then suggested that calculus be replaced by an algorithm which selects the best line from a set of random-generated lines. The proposal has been tested with an experimental algorithm written in Python language and found to work well.
\end{abstract}

Keywords: linear data-fitting, least square, line of best fit, minimization methods, random-generated numbers, Python.

\section{Introduction}

Recently the present author contributed with a preprint ${ }^{(1)}$ to the Center for Open Science - OSF showing simple improvements to the linear least-squares method. This centuriesold method is routinely used to find the best straight line to be fitted thru scattered experimental points.

Figure 1 shows the position of a generic experimental point $P_{i}\left(s_{i}, t_{i}\right)$ relative to the desired best line $t=x s+y$. Traditionally, the method takes into consideration the vertical distance $B_{i} P_{i}$ (along the $t$-axis) between each point and the line. This distance is called the error of point $P_{i}$. Each error is squared and summed for all experimental points, giving the total vertical squared error (VE). Unknown parameters ( $x=$ slope, $y=$ intercept $)$ of the "best" line are determined by searching for the minimum of total squared error. This is done with the tools of differential calculus.

But there is no unique best line. Different methods or algorithms lead to different candidates to be the best one.

In the paper mentioned above, it is shown that if the horizontal distance $A_{i} P_{i}$ (along the $s$-axis) is chosen in place of the vertical one, a different line is determined, with equal rights to be considered the best one. This method may, in turn, be called the horizontal squared error (HE) approach. Additionally, it is also shown that summing the vertical total squared error with the horizontal total squared error one gets a grand total squared error and therefore the corresponding new best line. This new line stays between the VE and the HE lines, it somehow corrects for the bias in those lines, and may well be considered the line of best fit. Since it corresponds to minimizing the sum of the hypotenuse $A_{i} B_{i}$ of triangle $A_{i} B_{i} P_{i}$, the approach is called the hypotenuse approach (HYE). 


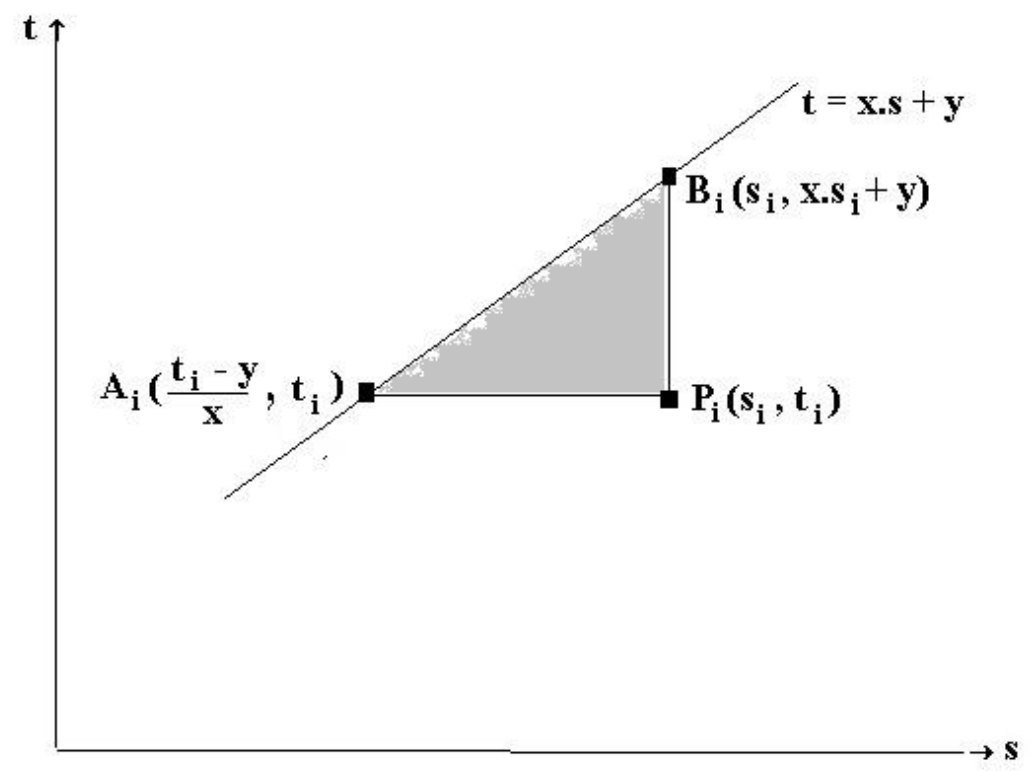

Figure 1 - Position of a generic experimental point $\mathrm{P}\left(\mathrm{s}_{\mathrm{i}}, \mathrm{t}_{\mathrm{i}}\right)$ relative to the best line $\mathrm{t}=\mathrm{xs}+\mathrm{t}$.

\section{Outliers are a problem}

Squaring individual errors avoids mutual cancellation. But it also distorts the force of attraction of each point on the line. Distant points (the outliers) exaggeratedly attract the line toward themselves. Vertical and horizontal approaches react differently to an outlier, and the hypotenuse approach may be more helpful in general to diminish the bias in using VE or HE only.

Then, squaring solves one problem but brings in a different one, one that may be serious in certain circumstances.

Of course, outliers may be left out, ignored, but they are also pieces of information with their own intrinsic value. Then the point is: keep the outliers, but do not let points attract the line quadratically with distance. A possible solution is the use of absolute values to avoid cancellation, instead of squaring.

One could choose to minimize the total error of type HYE since this itself is already better than VE or HE alone. Then function HYE written in terms of absolute values is:

$$
\mathrm{HYE}=\Sigma_{\mathrm{i}}\left|\mathrm{xs}_{\mathrm{i}}+\mathrm{y}-\mathrm{t}_{\mathrm{i}}\right|+\Sigma_{\mathrm{i}}\left|\left(\mathrm{t}_{\mathrm{i}}-\mathrm{y}\right) \mathrm{x}^{-1}-\mathrm{s}_{\mathrm{i}}\right|
$$

Summations are intended to be made over all experimental points $P\left(s_{i}, t_{i}\right)$. When function HYE is minimized, the values $(x, y)$ corresponding to the minimum will be the parameters $(x, y)$ of the line $t=x s+y$ of best fit.

Unfortunately, there exists no function capable of returning the absolute value of a number while also being everywhere differentiable. Then, in order to minimize the sum of the absolute errors, some tool other than differential calculus is required. 


\section{Testing the idea for feasibility}

Some tools are available, as an applet from a Wolfram Alpha package that succeeds in minimizing the function HYE of Eq. (1) and thus determines the line of best fit.

A different approach would be the use random numbers. Why not Monte Carlo or genetic algorithms? They certainly would do the job, but they also look too powerful tools for such a purpose.

Since the idea of using a random-powered algorithm seemed a good one and the function to minimize looked rather a simple one, the present author became tempted to write an experimental code in Python to test whether a random-number approach would work.

The idea behind the algorithm is simple: pairs of line parameters $(x, y)$ are sequentially chosen by a random generator and their resulting total errors are compared. If a pair gives a smaller error than the preceding one, it is kept; otherwise, it is abandoned. The process continues until a best-fit line deemed satisfactory is reached.

In order to start the investigation, Eq. (1) was rewritten as follows:

$$
\mathrm{HYE}=\Sigma_{\mathrm{i}}\left|\mathrm{xs}_{\mathrm{i}}+\mathrm{y}-\mathrm{t}_{\mathrm{i}}\right|+\Sigma_{\mathrm{i}}\left|\left[\left(\mathrm{t}_{\mathrm{i}}-\mathrm{y}\right)-\mathrm{xs}_{\mathrm{i}}\right] / \mathrm{x}\right|=\left(1+|\mathrm{x}|^{-1}\right)\left(\Sigma_{\mathrm{i}}\left|\mathrm{xs}_{\mathrm{i}}+\mathrm{y}-\mathrm{t}_{\mathrm{i}}\right|\right.
$$

And as a novelty, the absolute errors are raised to a power of exponent $p>0$, as shown in Eq, (2):

$$
H Y E=\left(1+|x|^{-p}\right) \Sigma_{i}\left|x s_{i}+y-t_{i}\right|^{p}
$$

Such an addition gives useful flexibility. If exponent $p$ is put at the value $p=2$, the new algorithm should repeat the results given by the traditional linear squared-error method, therefore affording an easy way to check the functionality of a new algorithm. And to use the absolute error methodology, one just keeps the exponent at $p=1$.

\section{Some results}

In the discussion to follow, the solutions (lines of best fit) offered by the random-based algorithm will be compared with those of classic square-error method and of the Alpha applet. The sets of experimental points taken from paper ${ }^{(1)}$ are presented in Table I and Table II. These Tables also show the best fit line, calculated according to the squarederror approach.

Table I - Experimental points; line of best fit by a square-error

\begin{tabular}{|c|c|c|c|c|c|c|c|}
\hline $\mathbf{S}_{\mathbf{i}}$ & 1 & 2 & 3 & 4 & 5 & 6 & line of best fit: \\
\hline $\mathbf{t}_{\mathbf{i}}$ & 5 & 6 & 4 & 4 & 10 & 12 & $t=1.61 \mathrm{~s}+1.21$ \\
\hline
\end{tabular}
method according to the HYE approach 
Table II - Experimental points with distant outlier; line of best fit by a square-error method according to the HYE approach

\begin{tabular}{|c|c|c|c|c|c|c|c|}
\hline $\mathbf{S}_{\mathbf{i}}$ & 1 & 2 & 3 & 4 & 5 & 6 & line of best fit: \\
\cline { 1 - 6 } $\mathbf{t}_{\mathbf{i}}$ & 1 & 2 & 3 & 20 & 5 & 6 & $t=2.32 s-1.95$ \\
\hline
\end{tabular}

Table III shows how the three methods (square-error, random-based, Alpha) compare in minimizing the same HYE function with a specified value of exponent $p$.

As far as the random algorithm is concerned, the rectangle where the random generator extracts all pairs $(x, y)$ is determined by vertices: $(-20,-30),(-20,20),(30,20),(30,-30)$. The algorithm needs an initial line to start, and this line is chosen to be $t=s$ (a 45degrees line thru the origin of axes).

Table III - Comparison of results

\begin{tabular}{|l|c|c|c|c|}
\hline $\begin{array}{c}\text { Source } \\
\text { of data }\end{array}$ & $\begin{array}{c}\text { Value of } \\
\text { exponent } \mathbf{p}\end{array}$ & $\begin{array}{c}\text { Line of best fit, by } \\
\text { squared error }\end{array}$ & $\begin{array}{c}\text { Line of best fit, by } \\
\text { random algorithm }\end{array}$ & $\begin{array}{c}\text { Line of best fit, by } \\
\text { Wolfram Alpha }\end{array}$ \\
\hline Table I & 2 & $\mathrm{t}=1.61 \mathrm{~s}+1.21$ & $\mathrm{t}=1.59 \mathrm{~s}+1.27$ & $\mathrm{t}=1.61 \mathrm{~s}+1.21$ \\
\hline Table I & 1 & - & $\mathrm{t}=1.36 \mathrm{~s}+3.22$ & $\mathrm{t}=1.33+3.33$ \\
\hline Table II & 2 & $\mathrm{t}=2.32 \mathrm{~s}-1.95$ & $\mathrm{t}=2.31 \mathrm{~s}-1.92$ & $\mathrm{t}=2.32 . \mathrm{s}-1.95$ \\
\hline Table II & 1 & - & $\mathrm{t}=1.13 \mathrm{~s}-0.30$ & $\mathrm{t}=1.13 \mathrm{~s}-0.33$ \\
\hline
\end{tabular}

In the first row of Table III, the set of experimental points is the one in Table I, and $p=$ 2. In this case, square-error and Alpha algorithms agree entirely, with random algorithm getting very close to them. The conclusion is that a random-based method appears to be reliable.

The second row in Table III shows the results for $p=1$. A surface plot of the error as a function of parameters $(x, y)$ is shown on Fig.2. The figure shows the singularity at $\mathrm{x}=0$ where the horizontal error HE has no meaning. The rest of the surface is smooth. It is the minimum of this surface that is sought, whose coordinates ( $x=$ slope, $y=$ intercept $)$ are the parameters of the line of best fit.

Square-error methods do not work now. It is seen that Alpha confirms the line of best fit found by the random algorithm.

Notice that the lines of best fit in the first row are slightly different from those in the second row because they are obtained by different methodologies. In the first row, the experimental points attract the best line according to the square of the distance from it; in the second row, the attraction is done (perhaps more naturally) according to the distance.

The last two rows in Table III make use of the set in Table II, which contains a very distant outlier. The example has been constructed for the purpose of assessing the possible benefits from using the absolute value in place of the squaring. Here the benefits are great, as it may be seen by comparing the result in the third row to that in the fourth row. 


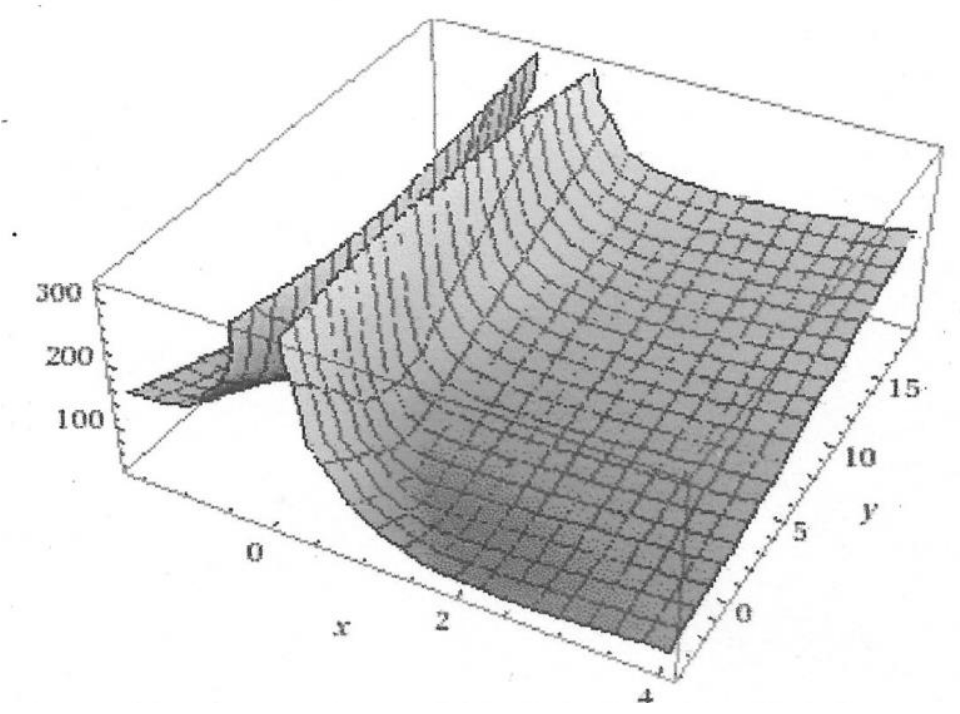

Figure 2 - Total error HYE (vertical axis $z$ ) is a function of $(x, y)$, respectively slope and intercept of lines, for the case of Table II (a distant outlier is present) and $\mathrm{p}=1$ (use of absolute values of error).

\section{Details of a specific case}

In the following pages, the case in the third row of Table III is presented with some details to earn a better knowledge of how a random-based algorithm works. The case was chosen because there is an outlier among the experimental points and the outcome of the random algorithm is confirmed by both the square-error method and Alpha.

Fig. 3 informs on how the algorithm starts from a large initial error and after nine successful attempts gives the line of best fit. The process has low efficiency: only nine successes in 500,000 trials!

All experimental points are shown on Fig.4. The outlier is clearly identified and the influence of exponent $p$ may visually be evaluated. The line corresponding to $p=2$ is the closest to the outlier but when $p=1$ the effect of the outlier is greatly neutralized. The case with the intermediate value $p=1.2$ has been included to show how gradually decreasing $p$ moves the line farther from the outlier.

Additionally, if one makes $0<p<1$, the power of attraction of points becomes even lower.

Finally, Fig. 5 offers a three-dimensional rendering of the convergence path: a zigzag line from the point corresponding to the initial values of (x,y), marked as "start", up to the final result marked as "finish". 
G. Gambirasio - Proposal for a Random-based Algorithm

to Solve Data-fitting Problems

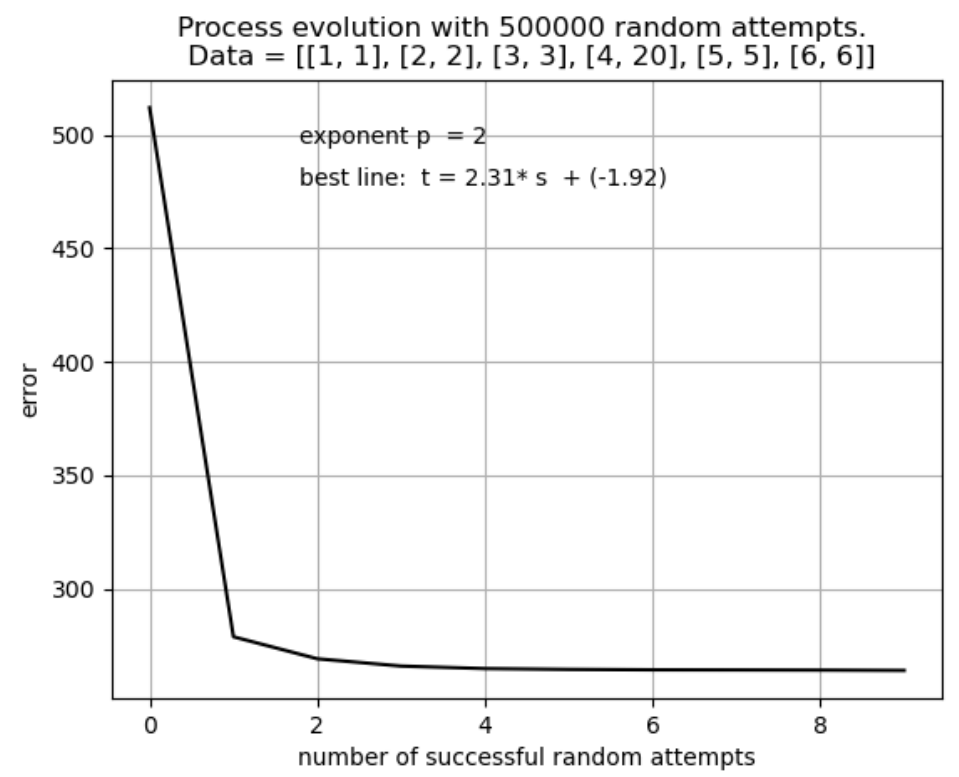

Figure 3 - In the random algorithm, the quadratic error diminishes in correspondence of each of the nine successful attempts. The figure shows how many pairs $(x, y)$ are given by the random generator $(500,000$ pairs); the coordinates of experimental points; the exponent $(p=2)$; and the final best line.

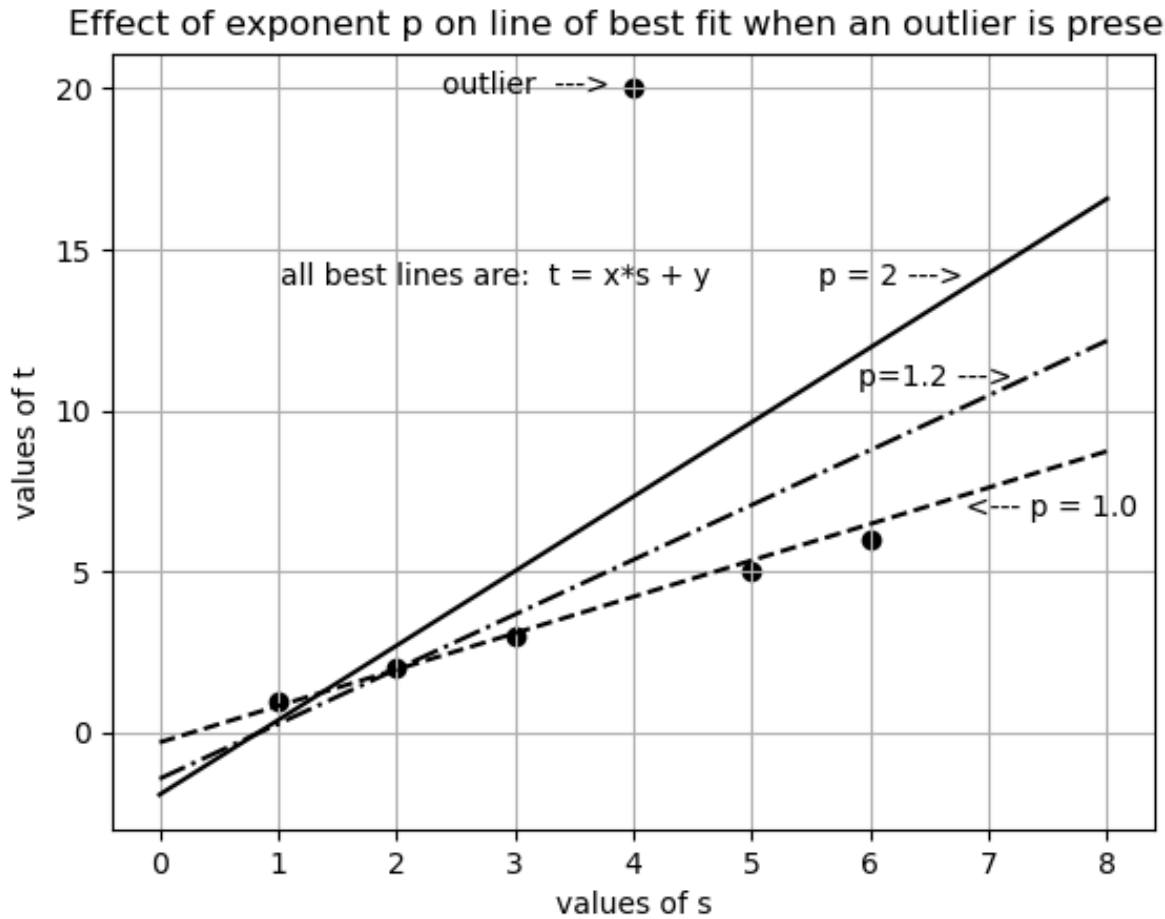

Figure 4 - The experimental points of Table II are represented by round dots; the outlier is identified. Three best lines are presented, each one corresponding to a different value of exponent $p$. 


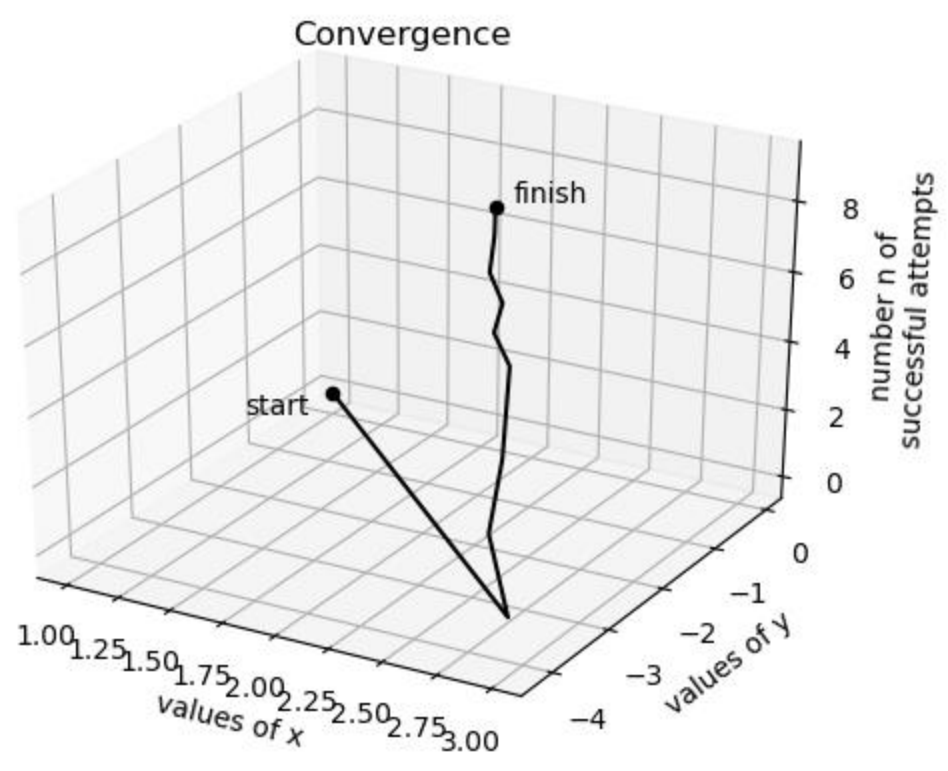

Figure 5 - A three-dimensional rendering of the zigzag path made by the random algorithm, from the initially given pair to the final result. Vertical axis $z$ refers to the numbered successful attempts.

\section{Conclusions}

Squaring errors makes experimental points attract the best line with a force proportional to the square of their distance from the line, therefore outliers exaggeratedly attract the line. Using absolute values instead of squaring would be a more natural procedure, but it prevents the utilization of differential calculus.

It is then suggested that calculus be replaced by an algorithm which selects the best line from a set of randomly generated lines. The proposal for a random-based algorithm tested satisfactorily with an experimental code written in Python language.

In the study, it has been added the possibility of raising the absolute errors to an arbitrary p-th power $(p>0)$. Exponent $p$ establishes the "power of attraction" of points on the best line and it is useful means to study the effect of outliers. Letting $p=2$ corresponds to the usual squaring error approach; $p=1$ corresponds to the case of absolute errors.

\section{References}

1 - Gambirasio, G., 'Comment on linear data-fitting methods', osf.io/56w7k 